\title{
THE ROLE OF ANTIBODIES IN DYSFUNCTION OF PIG-TO-BABOON PULMONARY TRANSPLANTS
}

Christine L. Lau, MD

William C. Daggett, MD ${ }^{\mathrm{a}}$

Mark F. Yeatman, FRCS ${ }^{a}$

Paul Chai, MD

Shu S. Lin, MD

Andrew J. Lodge, MD

Edward P. Chen, MD

Lisa E. Diamond, $\mathrm{PhD}^{\mathrm{b}}$

Guerard W. Byrne, $\mathrm{PhD}^{\mathrm{b}}$

John S. Logan, $\mathrm{PhD}^{\mathrm{b}}$

William Parker, $\mathrm{PhD}^{\mathrm{a}}$

Jeffrey L. Platt, MD

R. Duane Davis, MD
Objective: Pulmonary transplantation has become the preferred treatment for end-stage lung disease, but application of the procedure is limited because of a paucity of donors. One way to solve donor limitations is to use animal organs as a donor source or xenotransplantation. The current barrier to pulmonary xenotransplantation is the rapid failure of the pulmonary xenograft. Although antibodies are known to play a role in heart and kidney xenograft rejection, their involvement in lung dysfunction is less defined. This project was designed to define the role of antibodies in pulmonary graft rejection in a pig-to-baboon model.

Methods: Orthotopic transgenic swine left lung transplants were performed in baboons depleted of antibodies by one of three techniques before transplantation: (1) ex vivo swine kidney perfusion, (2) total immunoglobulindepleting column perfusion, and (3) ex vivo swine lung perfusion. Results were compared with those of transgenic swine lung transplants in unmodified baboons.

Results: All three techniques of antibody removal resulted in depletion of xenoreactive antibodies. Only pretransplantation lung perfusion improved pulmonary xenograft function compared with lung transplantation in unmodified baboons.

Conclusions: The pathogenesis of pulmonary injury in a swine-to-primate transplant model is different from that in renal and cardiac xenografts. Depletion of antibodies alone does not have a beneficial effect and may actually be detrimental. (J Thorac Cardiovasc Surg 2000;120:29-38) ung allotransplantation has become the preferred Ltreatment for end-stage pulmonary disease. The number of pulmonary transplants has reached a plateau of 1200 per year over the past 3 years. ${ }^{1}$ This plateau is not because the need is fulfilled, but because of the

From the Department of Surgery, ${ }^{a}$ Duke University Medical Center, Durham, NC; the Departments of Surgery, Pediatrics, and Immunology, ${ }^{c}$ Mayo Foundation, Rochester, Minn; and Nextran, ${ }^{\mathrm{b}}$ Princeton Forrestal Center, Princeton, NJ.

Supported by National Institutes of Health grants HL50985 and HL52297 and by Nextran. Christine L. Lau is a recipient of the International Society for Heart and Lung Transplantation Research Fellowship.

Received for publication April 1, 1999; revisions requested May 11, 1999; revisions received Feb 3, 2000; accepted for publication Feb 28, 2000.

Address for reprints: R. Duane Davis, MD, Department of General and Thoracic Surgery, Box 3864, Duke University Medical Center, Durham, NC 27710 (E-mail: davis053@mc.duke.edu).

Copyright () 2000 by The American Association for Thoracic Surgery

0022-5223/2000 $\$ 12.00+0 \quad \mathbf{1 2 / 1 / 1 0 6 8 4 1}$

doi: $10.1067 / \mathrm{mtc} .2000 .106841$ shortage of available and suitable donors. ${ }^{2}$ Additionally, because the lung is more susceptible than other organs to antemortem deterioration, even fewer lungs are available from the already limited number of human donors. One approach to extending transplantation as a therapy to all who need it is to use animals in lieu of human beings as a source of lungs, that is, xenotransplantation.

The major hurdle to clinical xenotransplantation is the severe immune reaction of the recipient against the graft. This reaction as it occurs in the xenografted heart and kidney has been extensively studied in recent years. To a certain extent, these studies have elucidated the pathogenesis of xenograft rejection at a molecular level and, as a result, have led to the development of incisive therapies. ${ }^{3-6}$ Thus, although some have speculated that clinical trials of heart and kidney xenografts might be in the offing, the current status of pulmonary xenotransplantation is less certain. Although pulmonary xenografts undergo functional deterioration and tissue injury at a tempo similar to that of rejection of cardiac and renal xenografts, the events leading to primary fail- 


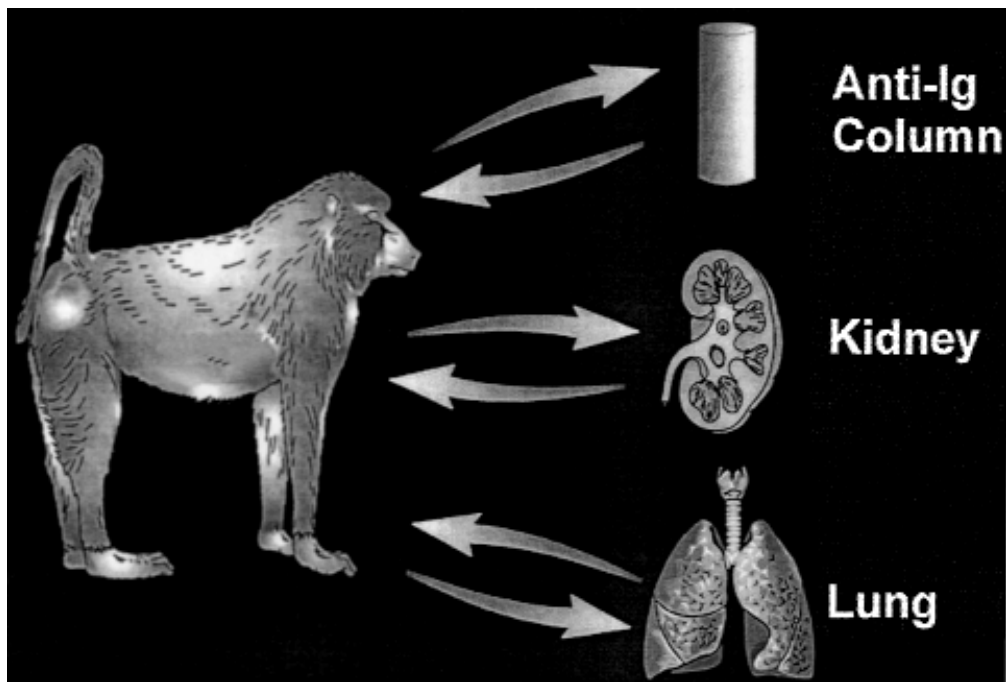

Fig 1. Schematic of pretransplantation antibody removal technique. This study comprised 4 groups. The control group consisted of transgenic swine lung transplants into unmodified baboons. $I g$, Immunoglobulin.

ure of lung xenotransplants are less certain. Complement has been shown to be important in acute lung dysfunction and injury. However, even when known defects in regulation of heterologous complement activation are corrected, a residual dysfunction remains in swine-to-primate lung transplants. ${ }^{7}$ Xenoreactive antibodies might play a role in this lung xenograft failure resulting from residual dysfunction. ${ }^{8-10}$ The goal of this article is to address the role of antibodies in residual lung xenograft dysfunction in an orthotopic pig-to-primate model.

\section{Materials and methods}

Animal care conformed to the standards of the National Society for Medical Research ("Principles of Laboratory Animal Care") and "The Guide for the Care and Use of Laboratory Animals" (NIH publication No. 86-23, revised 1985). Experiments were approved by the Duke University Institutional Animal Care and Use Committee.

Donor operation. Adult swine $(15-20 \mathrm{~kg})$ transgenic for human decay accelerating factor (hDAF) and CD59 were supplied by Nextran, Princeton, New Jersey. The constructs used in the generation of these swine contain a mouse $\mathrm{H}-2 \mathrm{~K}^{\mathrm{b}}$ promoter, regulating expression of complementary DNA for $\mathrm{hDAF}$, and a chick $\beta$-actin promoter controlling expression of a complementary DNA for human CD59. ${ }^{11}$

Animals were anesthetized with intramuscular administration of ketamine hydrochloride $(20 \mathrm{mg} / \mathrm{kg}$ ) and intravenous fentanyl $(100 \mu \mathrm{g} / \mathrm{kg})$. Endotracheal intubation and ventilation were established with $100 \%$ oxygen. All swine received methylprednisolone $(8 \mathrm{mg} / \mathrm{kg}$ ) and indomethacin (INN: indometacin) $(1 \mathrm{mg} / \mathrm{kg}) 1$ hour before harvest. The harvest procedure was performed as previously described. ${ }^{9}$
Alprostadil (prostaglandin $\left.\mathrm{E}_{1}\right)(50 \mu \mathrm{g} / \mathrm{kg})$ was injected directly into the pulmonary artery, and heparin was administered intravenously $(500 \mathrm{U} / \mathrm{kg}) 10$ minutes before harvest. Modified Euro-Collins solution containing heparin (10 $\mathrm{U} / \mathrm{mL})$, papaverine hydrochloride $(0.1 \mathrm{mg} / \mathrm{mL})$, methylprednisolone $(1 \mathrm{mg} / \mathrm{mL})$, indomethacin $(0.1 \mathrm{mg} / \mathrm{mL})$, and nicardipine $(0.2 \mathrm{mg} / \mathrm{mL})$ (Wyeth Laboratories Inc, Philadelphia, Pa) was administered into the pulmonary artery from a height of $30 \mathrm{~cm}(25 \mathrm{~mL} / \mathrm{kg})$ for pulmonary preservation. The heart and lungs were then removed en bloc and immersed in cold $\left(4^{\circ} \mathrm{C}\right)$ saline solution.

Kidney harvest/ex vivo perfusion (Fig 1). Baboons underwent immunodepletion by ex vivo swine kidney perfusion $(n=3)$. Pigs used as kidney donors were hDAF/CD59. The harvest technique has been described previously. ${ }^{12}$ For each baboon $(n=3)$, intravenous cannulas were placed in the femoral artery and femoral vein under direct visualization, and after heparinization $(1000 \mathrm{U} / \mathrm{kg})$ they were connected to the renal artery and vein, respectively, of 4 swine kidneys. Each kidney was perfused for 30 minutes with only 1 kidney at a time being perfused (ie, in sequence). A flow probe was not used to assess perfusion through the kidneys.

Depletion of immunoglobulin (Fig 1). Immunoglobulin depletion was accomplished by separating plasma and passing it though anti-immunoglobulin columns (Therabsorb; Unterschleisshein, Germany) as previously described. ${ }^{4}$ With the baboons under general anesthesia, a splenectomy was performed and a double-lumen Hickman catheter (Bard Access Systems, Salt Lake City, Utah) was placed in the internal jugular veins. Immunoglobulin depletion was then performed 5 days before transplantation and on the morning of transplantation. Each treatment consisted of 8 to 12 cycles equaling approximately 3 to 5 plasma volumes. The animals were heparinized $(1000 \mathrm{U} / \mathrm{kg})$ for each column treatment. 
Pretransplantation extracorporeal pulmonary perfusion (Fig 1). Baboons underwent immunodepletion by ex vivo lung perfusion $(n=3)$ by the method previously described. ${ }^{9}$ All swine used as ex vivo lung donors were hDAF/CD59 transgenic animals. Baboons were heparinized before perfusion $(1000 \mathrm{U} / \mathrm{kg})$.

Pulmonary transplantation. Baboons $(12-16 \mathrm{~kg})$ were sedated with ketamine hydrochloride $(10 \mathrm{mg} / \mathrm{kg})$, intubated, and their lungs were ventilated with an adult volume-controlled ventilator (Bennett Respiration Products, Inc, Santa Monica, Calif) with $100 \%$ oxygen at a rate of 10 breaths/min with a tidal volume of $12 \mathrm{~mL} / \mathrm{kg}$. All baboons underwent left pneumonectomy followed by heparinization $(1000 \mathrm{U} / \mathrm{kg})$ and orthotopic swine lung transplantation as described previously. ${ }^{9}$

Immunosuppression. Baboons undergoing column absorption as the method of antibody depletion were immunosuppressed beginning the first day of antibody depletion (day -5). The immunosuppressive regimen consisted of methylprednisolone $(10 \mathrm{mg} / \mathrm{kg}$ per day tapered by $1 \mathrm{mg} / \mathrm{kg}$ per day, cyclosporine $(5 \mathrm{mg} / \mathrm{kg}$ per day after a loading dose of $15 \mathrm{mg} / \mathrm{kg}$ ), and cyclophosphamide $(1-5 \mathrm{mg} / \mathrm{kg}$ per day after a loading dose of $10 \mathrm{mg} / \mathrm{kg}$ per day for 2 to 3 days). ${ }^{3,4}$

Baboons undergoing kidney or lung perfusion as the method of antibody depletion received methylprednisolone (Solu-Medrol; $8 \mathrm{mg} / \mathrm{kg}$ ), cyclosporine (10-15 mg/kg), and azathioprine (Imuran; $2 \mathrm{mg} / \mathrm{kg}$ ) on the morning of the experiment.

Data acquisition. Pulmonary artery flow and cardiac output were measured with an ultrasonic flowmeter (Transonics Systems Inc, Ithaca, NY), and pulmonary artery pressure was determined with a Millar Mikro-Tip micromanometer (Millar Instruments, Inc, Houston, Tex) positioned in the proximal main pulmonary artery.

Analysis of tissue biopsy specimens. Biopsy specimens for hematoxylin and eosin staining were taken from varying areas of the lung and fixed in $10 \%$ buffered formalin for greater than 24 hours. After treatment with formalin, the samples were dehydrated and embedded in paraffin. The embedded samples were cut into 4- $\mu \mathrm{m}$ thick sections, rehydrated, and stained with hematoxylin and eosin.

For immunohistochemical studies, samples were obtained, frozen, sectioned, and fixed as previously described. ${ }^{4}$ Sections from each sample were incubated with affinity-isolated fluorescein isothiocyanate (FITC)-conjugated goat antihuman immunoglobulin (Ig) M ( $\mu$-chain specific; Kirkegaard \& Perry Laboratories, Inc, Gaithersburg, Md); affinity-isolated, FITC-conjugated goat anti-human IgG ( $\gamma$-chain specific; Kirkegaard \& Perry Laboratories, Inc); affinity-isolated, FITC-conjugated goat anti-human C3 (Organon TeknikaCappel, Durham, NC); affinity-isolated, FITC-conjugated goat anti-human C4 (Organon Teknika-Cappel); affinity-isolated, FITC-conjugated rabbit anti-human fibrinogen (Accurate Chemical and Scientific Corp, Westbury, NY); murine monoclonal antibody against human C5b neoantigen (Quidel, San Diego, Calif); or murine monoclonal antibody against a neoantigen of the membrane attack complex (MBM5; generously provided by A. F. Michael, University of
Minnesota, ${ }^{13}$ as previously described $\left.{ }^{14}\right)$. Tissue sections were then washed with phosphate-buffered saline solution. Unlabeled murine monoclonal antibodies were detected with a double fluorochrome antibody layer consisting of affinityisolated, $\mathrm{F}\left(\mathrm{ab}^{\prime}\right) 2$ FITC-conjugated goat anti-mouse $\operatorname{IgG}$ and affinity-isolated $\mathrm{F}\left(\mathrm{ab}^{\prime}\right) 2$ FITC-conjugated rabbit anti-goat IgG (Organon Teknika-Cappel). After the staining procedures, tissue sections were washed with phosphate-buffered saline solution and mounted with $p$-phenylenediamine/glycerol solution. ${ }^{14}$ All anti-human reagents were shown to cross react with their baboon counterparts. Background immunofluorescence was assessed by omitting the primary antibodies. The tissue samples were studied with a Leitz DMRB epifluorescence microscope (Leitz, Wetzlar, Germany).

Quantitation of total IgM and IgG. Total immunoglobulin levels were determined by an enzyme-linked immunosorbent assay with the use of affinity-purified alkaline phosphatase-conjugated goat antibodies specific for human $\mu$ chain or $\gamma$-chain (Sigma Chemical Co, St Louis, Mo), as previously described. ${ }^{4}$ The assays were carried out at room temperature and the absorbance at $405 \mathrm{~nm}$ was determined with an EL 340 Bio Kinetics Reader (Bio Kinetics Corp, San Antonio, Tex).

Quantitation of xenoreactive IgM and anti-galactose $\alpha(1,3)$ galactosyl (anti-Gal $\alpha[1,3]$ Gal) antibody levels. The levels of xenoreactive antibodies present in the serum or plasma samples taken during the experiment were determined on the basis of binding to cultured porcine aortic endothelial cells, as described previously. ${ }^{15}$ Comparison with serum containing known levels of anti-Gal $\alpha(1,3) \mathrm{Gal} \mathrm{IgM}$ was made to determine absolute levels. ${ }^{16,17}$

Statistical analysis. Values are reported as mean \pm SEM and analyzed by 1-way analysis of variance with comparisons between the groups made with the Student-Newman-Keuls test. Data were analyzed with Glantz's primer of biostatistics computer software (version 4.02, McGraw-Hill, 1996).

Termination of experiments. Per protocol, these studies were terminated at 24 hours or sooner if graft flow was less than $50 \mathrm{~mL} / \mathrm{min}$ for 2 consecutive hours.

\section{Results}

Four lungs from swine expressing hDAF and human CD59 were transplanted into baboons. Results previously reported have showed that 1-hour blood flow though these transgenic lungs was 12-fold (317.5 \pm $61.4 \mathrm{~mL} / \mathrm{min}$ ) the rate through unmodified swine lungs (26.7 $\pm 12.1 \mathrm{~mL} / \mathrm{min})$. However, blood flow was still only $22 \%$ of the cardiac output. Immunohistochemical staining of the lung tissues did not show significant deposition of IgM, C3, C4, and membrane attack complex within the graft microvasculature. In lung transplants performed with outbred swine as donors, early pulmonary edema and microvascular thrombosis were seen. Among the 4 transgenic swine lungs expressing hDAF and CD59, 2 had moderately severe pulmonary 


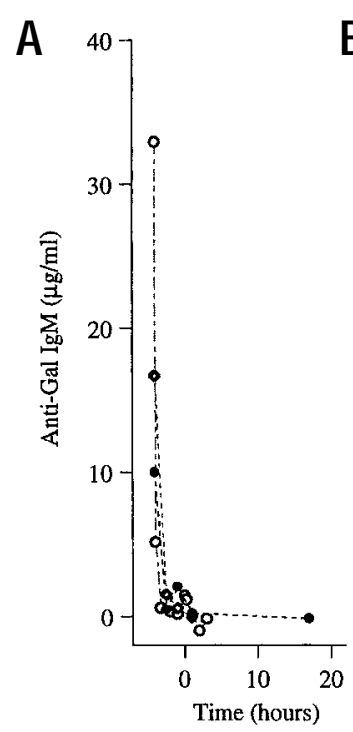

Kidney

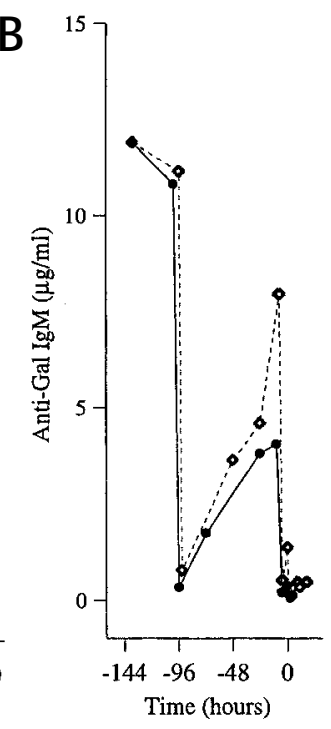

Ig-depleting column

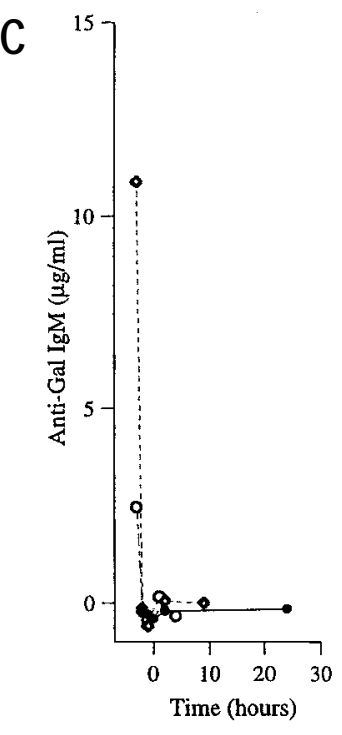

Lung

Method of Antibody Depletion

Fig 2A. Blood was obtained from each baboon recipient after pretransplantation antibody depletion (by perfusion through porcine kidneys $[A]$, Ig-depleting column $[B]$, or porcine lungs $[C]$ ) and after transplantation of a transgenic porcine lung expressing human decay accelerating factor (hDAF) and CD59. The level of anti-Gal $\alpha(1-3) \mathrm{Gal}$ IgM was measured by enzyme-linked immunosorbent assay on cultured porcine endothelial cells from the binding eliminated by treatment of the cells with $\alpha$-galactosidase. Absolute concentrations were determined after comparison with a referenced standard. Time 0 refers to the time of transplantation. The group undergoing pretransplantation antibody depletion by Ig-depleting column underwent two separate column treatments (day -5 and day 0 ) with a rebound seen after the first treatment. The difference in remaining anti-Gal $\alpha(1-3)$ Gal antibodies after each pretransplantation depletion technique was not significant between the treated groups.

1

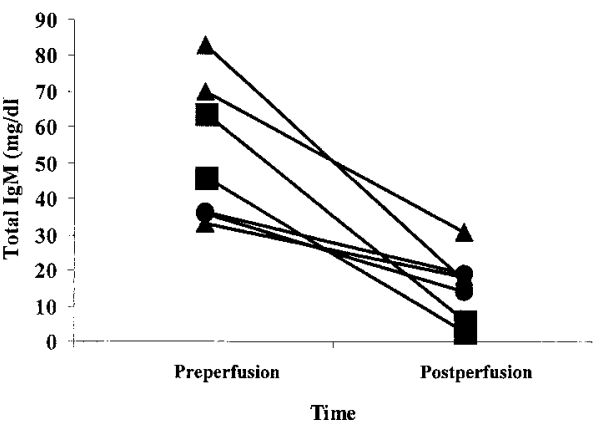

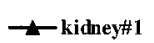
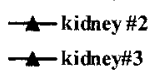

-7 anti-Ig\#1

- - anti-Ig\#2

$\rightarrow$ lung\#1

$\rightarrow$ lung\#2

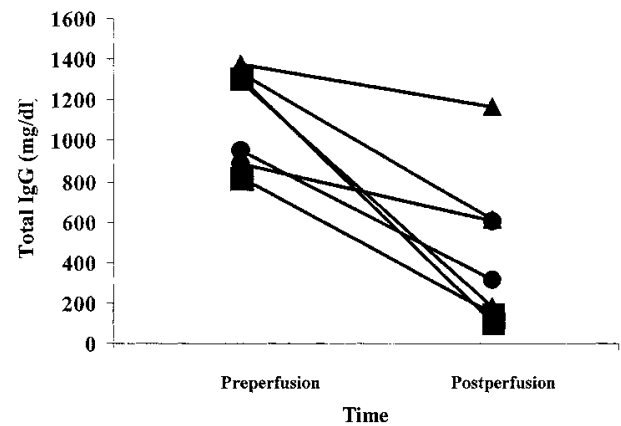

Fig 2B. The total $\operatorname{IgM}(1)$ and $\operatorname{IgG}(2)$ antibodies remaining after pretransplantation antibody depletion by kidneys, total immunoglobulin-depleting columns, or lungs as measured by enzyme-linked immunosorbent assay. After pretransplantation perfusion with either kidneys or lungs, total immunoglobulin in the serum was modestly lower than baseline compared with the profound decrease after total immunoglobulin-depleting column perfusion.

edema associated with alveolar wall disruption and all 4 had fibrin plugs apparent at 180 minutes. These results have been reported previously. ${ }^{7}$

To test whether the residual defect in pulmonary function in transgenic lungs was caused by xenoreac- tive antibodies, we assessed whether depletion of xenoreactive natural antibodies would prevent physiologic and pathologic deterioration of the lung xenografts. Xenoreactive antibody depletion was accomplished by perfusion of blood of the baboon 

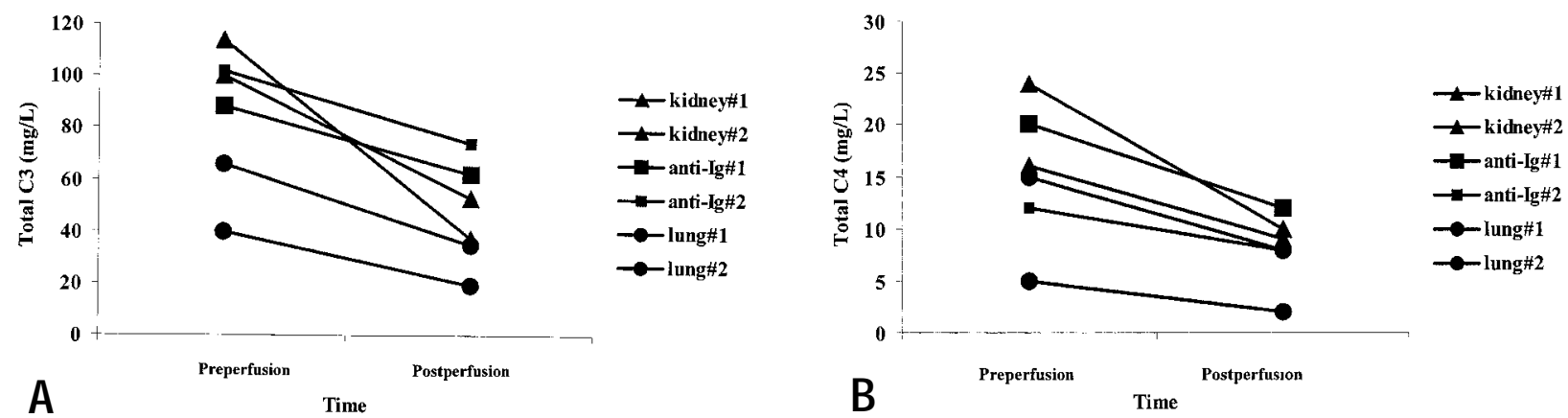

Fig 3. Percentage of total C3 (A) and C4 (B) levels remaining after each depletion technique. There was not an appreciable difference in remaining total complement levels between treated groups.

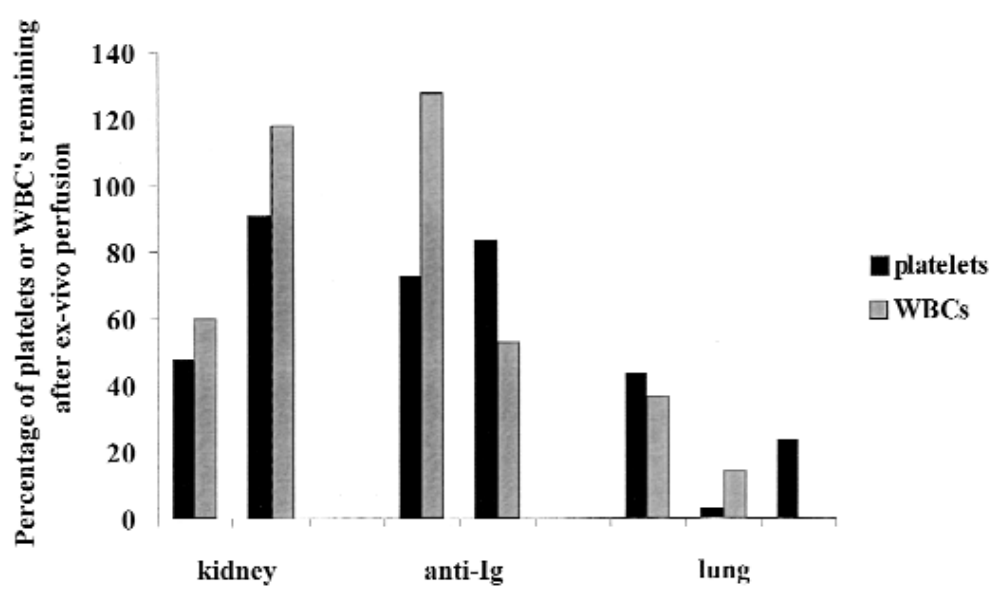

Experimental Group

Fig 4. Percent of total number of platelets or WBCs remaining after each depletion technique for each animal. Note that ex vivo lung perfusion results in substantially more depletion of platelets and WBCs than the other techniques.

through 4 pig kidneys, and the results are shown in Figs $2 \mathrm{~A}$ and $2 \mathrm{~B}$. The degree of baboon complement, platelet, and white blood cell (WBC) depletion by ex vivo kidney perfusion is shown in Figs 3 and 4. As shown in Fig 5, A, not only did depletion of xenoreactive antibodies by perfusion of porcine kidneys fail to improve blood flow in the transplanted transgenic swine lung, it seemingly worsened flow, with graft flows at 1 hour only $50 \%$ of graft flows in transgenic swine not undergoing depletion of xenoreactive antibodies. Immunohistochemical staining of biopsy specimens from the transgenic swine lung transplanted into baboons after undergoing xenoreactive natural antibody depletion by perfusion with swine kidneys failed to show significant deposition of $\mathrm{IgM}, \mathrm{C} 3, \mathrm{C} 4$, and membrane attack complex within the graft microvasculature and only trace deposition in the larger pulmonary arteries. In contrast, in the transgenic porcine kidneys used to deplete xenoreactive antibodies, IgM, C3, C4, and membrane attack complex deposition was strongly positive (Fig 6). Histopathologic examination of the ex vivo perfused kidneys revealed microvascular thrombi and edema (Fig 7, A). The 1-hour post-transplantation lung biopsy specimens from the group with pretransplantation kidney depletion were relatively unremarkable (Fig 7, B), but by the time of death of the baboons (24 hours) the microscopic appearance of the lungs was significant for increased cellularity, hemorrhage, microvascular thrombosis, and edema (Fig 7,C).

We wished to test whether depletion of xenoreactive antibodies by pretransplantation kidney perfusion might have failed to remove certain immunoglobulins specifically directed against the pulmonary vasculature from the circulation of the baboons. Therefore, we performed 

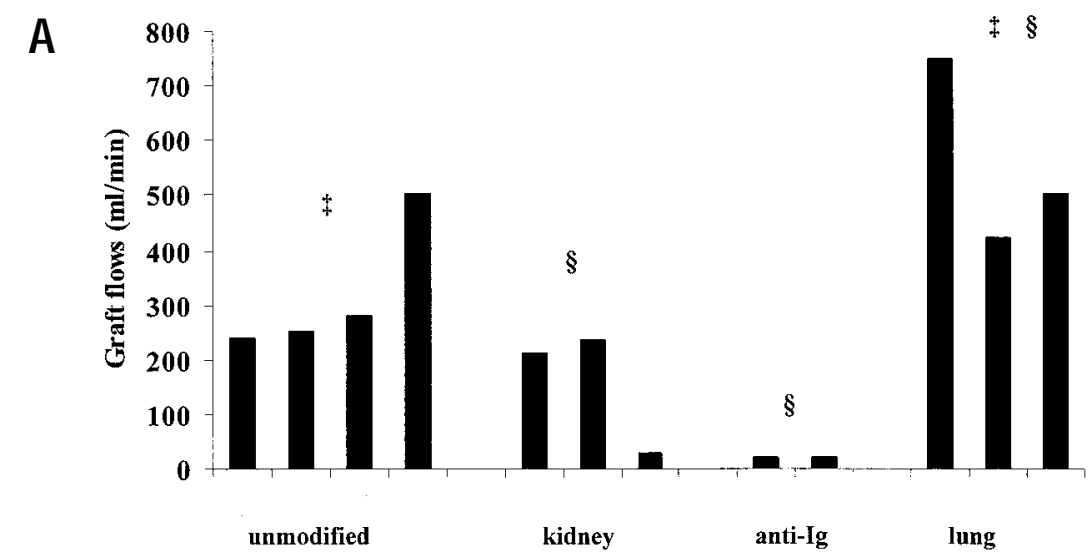

B

Experimental Group

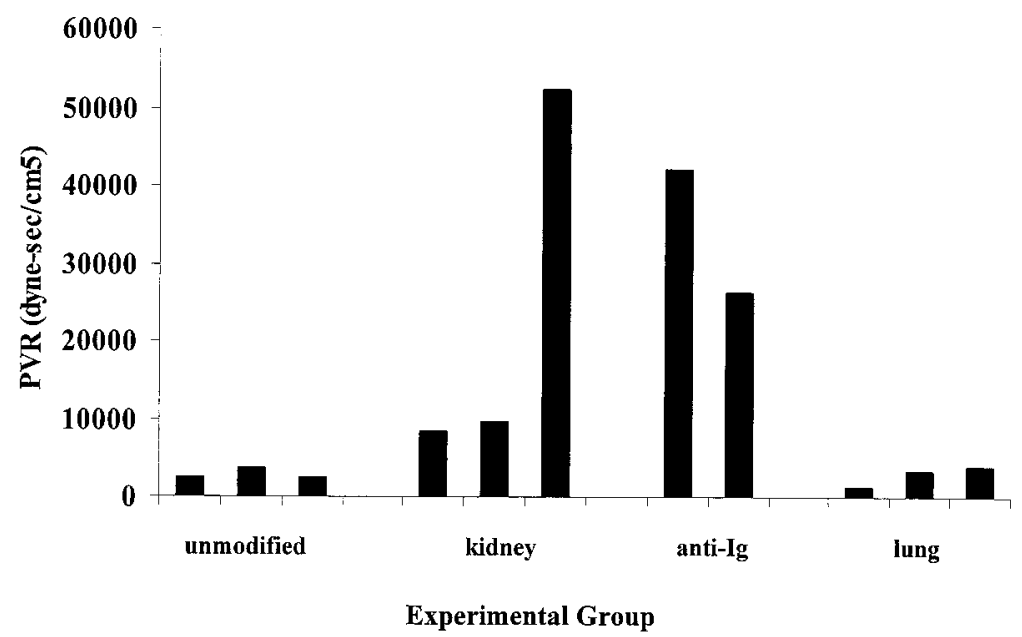

Fig 5. Physiologic variables of pulmonary xenografts. Measurements were made after the recipient's blood pressure and heart rate had stabilized, between 15 and 60 minutes after reperfusion. ${ }^{\ddagger} P<.05$ between control and lung group; ${ }^{\S} P<.05$ between lung and anti-immunoglobulin column groups and between lung and kidney groups. A, Lung xenograft flows. B, Pulmonary vascular resistance $(P V R)$ across xenograft.

a series of experiments in which blood from baboons was depleted of all immunoglobulins by passage of plasma through columns bearing anti-human immunoglobulin antibodies as recently described. ${ }^{4}$ IgM specific for Gal $\alpha(1,3) \mathrm{Gal}$ and total IgM and $\mathrm{IgG}$ antibody remaining after column depletion are shown in Figs 2A and 2B. Depletion of total IgM and IgG to less than 20\% (6.3\%9.6\% IgM; $7.9 \%-18.5 \%$ IgG) was achieved. The degree of baboon complement, platelet, and WBC depletion by pretransplantation column perfusion is shown in Figs 3 and 4. Despite effective depletion of the immunoglobulins, the porcine pulmonary xenografts had flows at 60 minutes that were $6.3 \%$ of transgenic swine lungs transplanted in unmodified baboons (Fig 5, A). The porcine lungs transplanted into immunoglobulin-depleted baboons had extensive microvascular thrombi throughout all lung fields at 60 minutes (Fig 7, D).
To further define the role of xenoreactive antibodies in pulmonary xenograft dysfunction, we performed a series of experiments in which blood from baboons was depleted of xenoreactive antibodies by perfusion through pig lungs. Presumably, perfusion of the porcine lungs allowed removal of conventional xenoreactive antibodies and antibodies specific for the lung. Anti-Gal $\alpha(1-3) \mathrm{Gal}$ xenoreactive antibodies were depleted (Fig 2A) after perfusion with swine lungs while total $\operatorname{IgM}$ and $\operatorname{IgG}$ only were moderately reduced (Fig 2B). The degree of baboon complement, platelet, and WBC depletion by ex vivo lung perfusion is shown in Figs 3 and 4. As seen in Fig 5, only pretransplantation lung perfusion significantly improved pulmonary xenograft flows, with a $175 \%$ increase in graft flow at 60 minutes compared with transgenic lungs transplanted into unmanipulated baboons. There was minimal if 

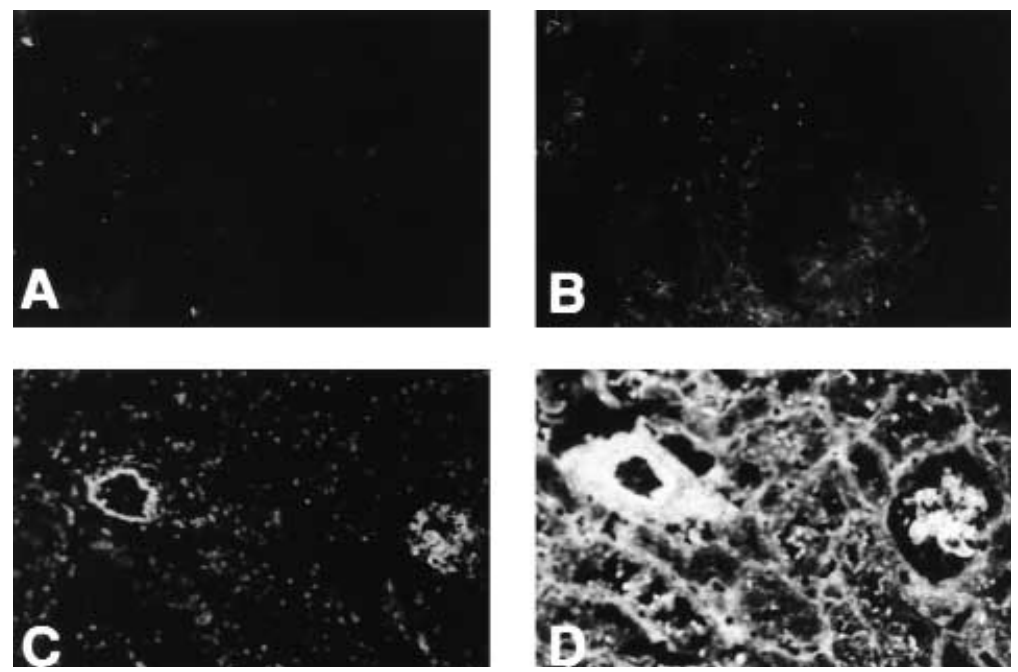

Fig 6. Deposition of IgM and IgG in pretransplantation-perfused porcine lungs or kidneys. Biopsy specimens from these pretransplantation-perfused organs were analyzed by immunofluorescence microscopy for deposition of IgM and IgG. A, IgM. B, IgG. Pretransplantation lung perfusion at end showing no detection of IgM and only trace $\operatorname{IgG}$ in microvasculature of lung. IgG deposition was mostly interstitial, suggesting nonspecific deposition in pretransplantation lung. C, IgM. D, IgG. Pretransplantation kidney perfusion at end showing prominent deposition of both along the endothelial lining of blood vessels in the pretransplantation kidneys.

any deposition of $\mathrm{IgM}, \mathrm{IgG}, \mathrm{C} 3$, or $\mathrm{C} 4$ in the pulmonary microvasculature of the ex vivo (Fig 6) or the transplanted lung. At 120 minutes, the ex vivo lung was notable for the presence of edema, hemorrhage, and increased cellularity (Fig 7, E). In contrast, the architecture of the transplanted lung remained fairly normal throughout the experiment (24 hours) (Fig 7, F and $G$ ).

\section{Discussion}

A porcine organ transplanted into an unmodified primate is subjected to hyperacute rejection. In heart and kidney xenotransplantation, inhibition of complement activation successfully prevents hyperacute rejection, leading to the next phase, acute vascular rejection, which occurs in the time frame of days. Removal of antibodies prevents acute vascular rejection in these organs. ${ }^{4}$ In lung xenografts, the early dysfunction that appears similar in time frame to the hyperacute rejection of other xenografts is only partially abrogated by complement control.

Work by Daggett, ${ }^{9}$ Pierson, ${ }^{8}$ Macchiarini, ${ }^{10}$ and their associates has suggested that antibodies play a role in acute xenograft lung dysfunction in both an in vivo and an ex vivo pig-to-primate model. Daggett and colleagues ${ }^{9}$ found removal of xenoreactive antibodies of the baboon by pretransplantation perfusion with a swine lung resulted in improved hemodynamic variables and graft survival. Pierson and colleagues ${ }^{8}$ used an ex vivo lung perfusion model in which human blood was perfused through a pig heart-lung block. Pulmonary vascular resistance increased and pulmonary edema developed rapidly with a median time to graft failure of 20 minutes. In that model, thermal inactivation of complement did not prevent the rise in pulmonary vascular resistance. Absorption of xenoreactive antibodies did prevent the rise in pulmonary vascular resistance, but only the combination of heat inactivation of complement and absorption of xenoreactive antibodies prolonged graft survival to that achieved with autologous perfusion.

Macchiarini and colleagues ${ }^{10}$ reported similar findings to our data presented here, but in an ex vivo model. In their study they perfused whole human blood through swine lungs, livers, or spleens before ex vivo perfusion of the blood through a swine lung. They also perfused swine lungs with human plasma that had been depleted of anti-Gal antibodies by in vitro column immunoabsorption of the antibodies. Only swine lung perfusion of human blood before ex vivo perfusion of another swine lung resulted in improved functional and histologic survival of the ex vivo lung. Western blot analysis of plasma samples showed that prior swine lung perfusion removed antibodies against non-anti-Gal proteins of low molecular weight that were not eliminated by the antiGal columns. Their conclusions suggested that non-antiGal antibodies removed only by prior swine lung perfu- 

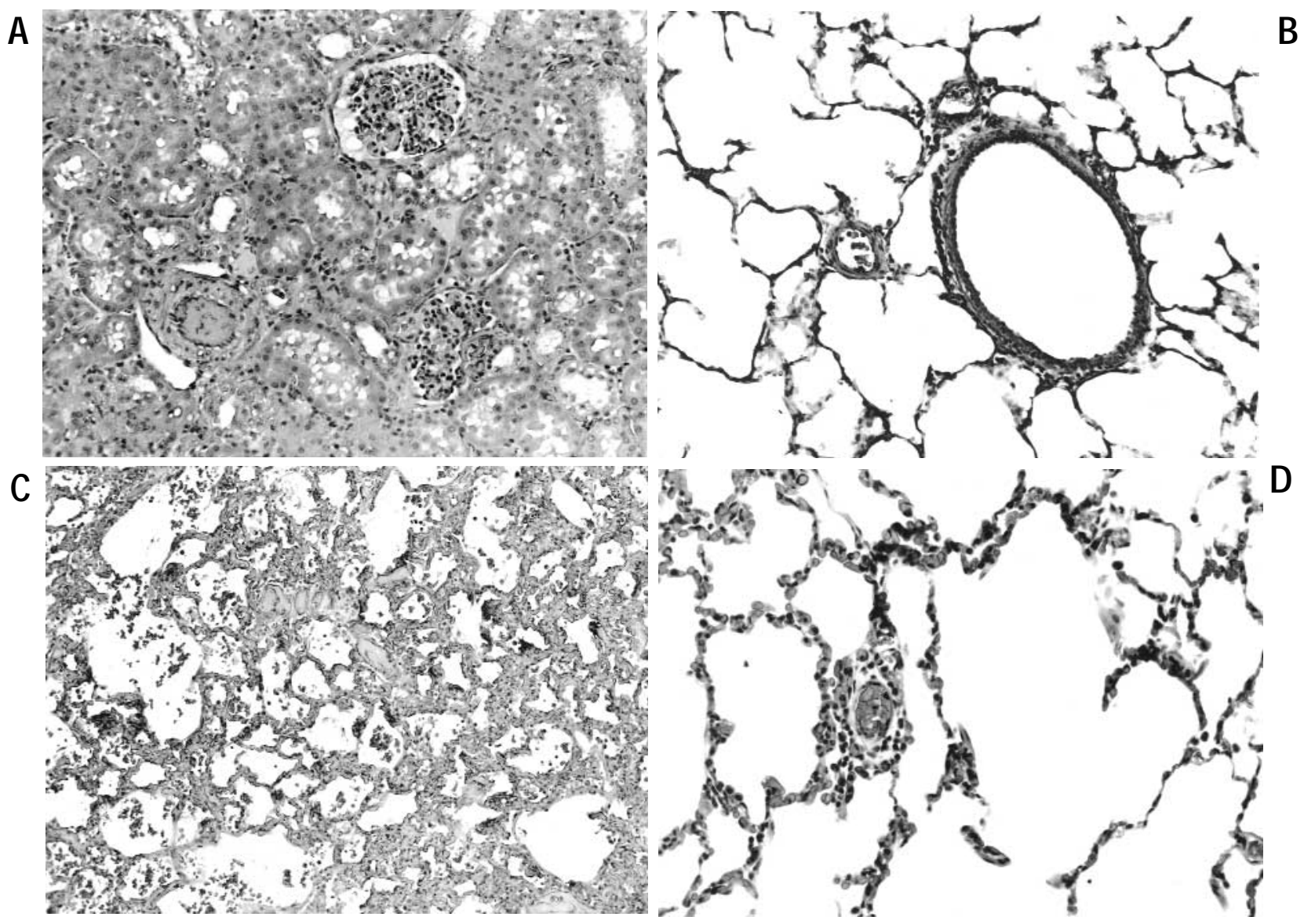

Fig 7. Histopathologic features in pretransplantation antibody depletion experiments. A to C, Pretransplantation kidney depletion experiments showing extensive microvascular thrombi and edema in ex vivo kidney (A), relatively unremarkable post-transplantation 1-hour biopsy (B), but increasing hemorrhage, cellularity, and edema with increasing time of transplantation (24 hours) (C). D, Extensive microvascular thrombi in 1-hour post-transplantation lung xenograft biopsy after pretransplantation treatment of baboon with total immunoglobulin-depleting column.

sion but not by liver, spleen, or column perfusion were the cause of improved survival of the ex vivo lungs.

Our data presented in this article support the value of preperfusion with a swine lung in lung graft survival, but our findings of worsening graft function with a significant reduction in total antibody levels by the antiimmunoglobulin columns are unexpected based on the data reported by Macchiarini and colleagues. ${ }^{10}$ Further evidence that preperfusion with swine lungs before swine lung transplantation is not beneficial because of removal of other non anti-Gal antibodies comes from our data in which we have looked for and have been unable to identify unique antigens on porcine pulmonary microvascular endothelial cells. ${ }^{18,19}$

Therefore, an important question emerging from our studies is the mechanism by which perfusion of the porcine lung confers a protective effect on subsequent lung xenografts. Depletion of circulating factors other than xenoreactive antibodies does occur with ex vivo lung perfusion. In addition to depleting xenoreactive antibodies, additional factors including platelets and WBCs are depleted when baboon blood is perfused through swine lungs. Platelets and WBCs have been shown by Pierson to play a role in pulmonary xenograft dysfunction in an ex vivo lung perfusion circuit (Pierson RN, personal communication). We have shown that swine von Willebrand factor is shed by lungs during perfusion with primate blood but not by kidneys (Platt JL, unpublished data). Swine von Willebrand factor in contrast to primate von Willebrand factor is capable of aggregating primate platelets in the absence of a cofactor or high shear stress. ${ }^{20}$ The throm- 


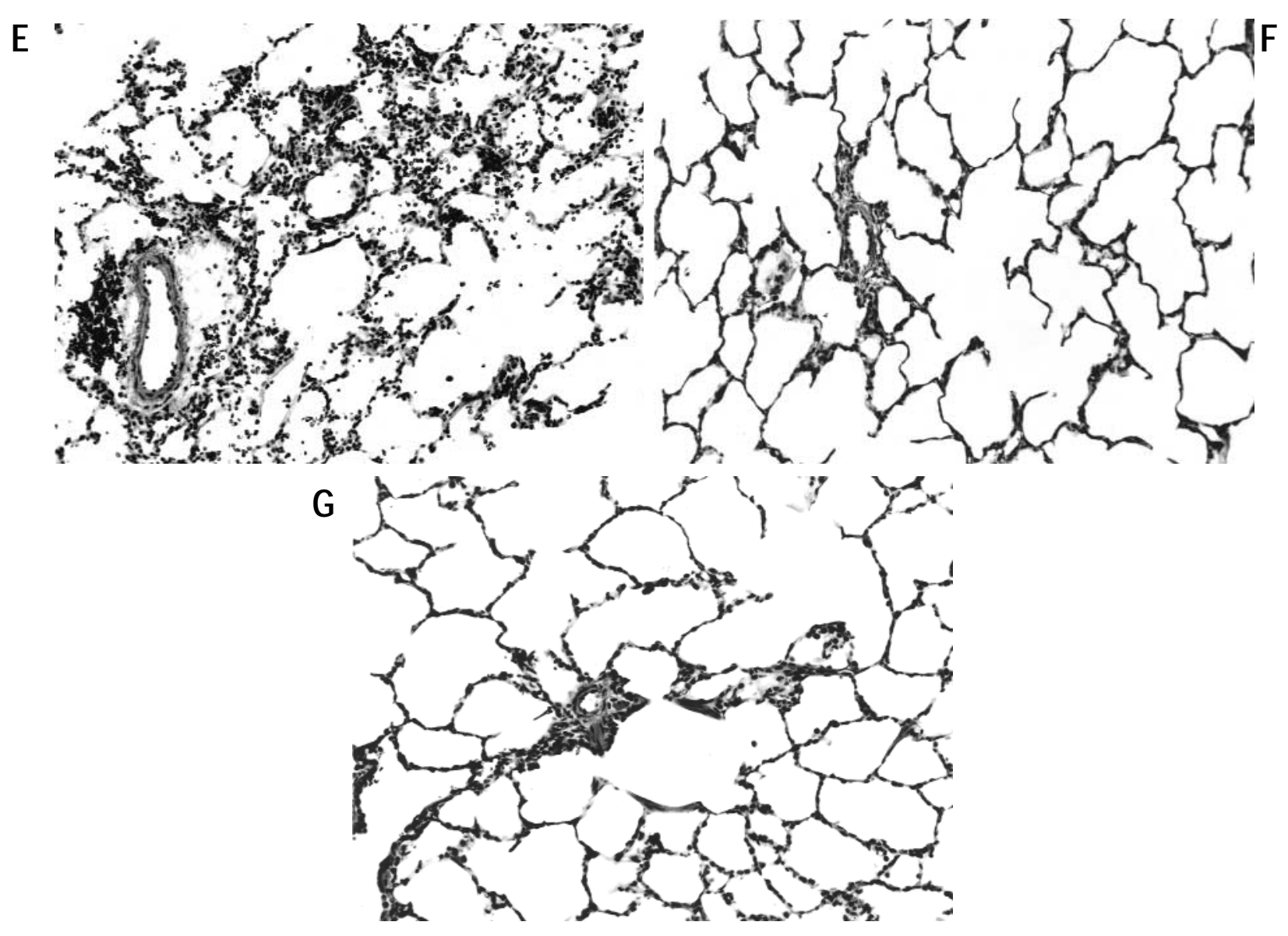

Fig 7. Cont'd. With the pretransplantation ex vivo lung after 120 minutes of perfusion with baboon blood, the presence of edema, hemorrhage, and increased cellularity is noted $(\mathbf{E})$, but the architecture of the transplanted lung remains fairly normal at 1-hour biopsy and throughout the experiment lasting 24 hours (F and $\mathbf{G})$.

bocytopenia occurring after lung perfusion may be the result of deposition of platelets (as a consequence of von Willebrand factor release) in the ex vivo organ, resulting in subsequent protection of the lung xenograft from platelet thrombi. Since swine von Willebrand factor is not released in substantial quantities with perfusion of swine kidneys, this protective effect (via platelet removal) is not appreciated. Swine von Willebrand factor would still be released by the transplanted lung, but the microvascular thrombi would be less in the preperfused swine lung group because of thrombocytopenia.

Although this discussion may explain why the xenografts transplanted into baboons after ex vivo lung perfusion have better function than the xenografts transplanted into unmodified baboons, it may be more difficult to explain why xenografts transplanted into baboons after ex vivo kidney perfusion or treatment with anti-immunoglobulin column have worse function than the xenografts transplanted into unmodified baboons. It is possible that the ex vivo swine kidney perfusion and anti-immunoglobulin column treatment of baboons removes protective factors or causes release of factors detrimental to the swine lung xenograft (but not to kidney or heart xenografts). For example, it has been proposed that the lung xenograft is more sensitive to small amounts of C3a and C5a (Platt JL, unpublished data) ${ }^{21}$ and thromboxane $\mathrm{A}_{2},{ }^{22}$ which may be released during ex vivo kidney perfusion or column absorption. Further studies are needed to test whether specific antibody depletion techniques are more toxic to lung xenografts. The severe microvascular thrombosis seen in the group treated with anti-immunoglobulin depleting column may be explained by the depletion of various anticoagulant proteins shifting the environment to a more procoagulant one. 
Swine von Willebrand factor is known to have Gal $\alpha(1,3)$ Gal epitopes and has been shown to bind anti-Gal $\alpha(1,3)$ Gal antibody. ${ }^{23}$ We have preliminary evidence that binding of anti-Gal $\alpha(1,3) \mathrm{Gal}$ antibodies to swine von Willebrand factor inhibits its ability to aggregate human platelets to a modest degree (Posther $\mathrm{KE}$, unpublished data). Therefore, it is possible that pretransplantation removal of antibodies in association with release of substantial swine von Willebrand factor from the lung xenograft results in accelerated microvascular thrombosis in the transplant. When unmodified baboons undergo swine lung transplants, the swine von Willebrand factor that is released complexes with anti-Gal antibodies, and its ability to aggregate primate platelets is modestly decreased, resulting in slightly improved function of the grafts.

In conclusion, the pathogenesis of pulmonary injury in swine-to-primate transplant model is different from that of heart and kidney. Antibody plays a complex role in acute injury of pulmonary xenografts. Removal of antibodies alone before transplantation does not appear to be beneficial and may actually be detrimental to the lung xenograft. Although pretransplantation lung perfusion depletes xenoreactive antibodies, additional factors including WBCs and platelets are depleted, which may explain the protective effect on the pulmonary xenograft. Although pretransplantation lung perfusion removes xenoreactive antibodies, additional factors including WBCs and platelets are also depleted. The depletion of these additional factors may explain the protective effect of pretransplantation lung perfusion on the lung xenograft.

We are indebted to George Quick, Kurt Campbell, Ronnie Johnson, and Robert McCall for their assistance during operative procedures.

\section{REFERENCES}

1. Hosenpud JD, Bennett LE, Keck BM, Fiol B, Boucek MM, Novick RJ. The Registry of the International Society for Heart and Lung Transplantation: Fifteenth official report-1998. J Heart Lung Transplant 1998;17:656-68.

2. Orians CE, Evans RW, Ascher NL. Estimates of organ specific donor availability for the United States. Transplant Proc 1993;25:1541-2.

3. Lin SS, Kooyman DL, Daniels LJ, et al. The role of natural antigal (1-3) gal antibodies in hyperacute rejection of pig-to-baboon cardiac xenotransplants. Transplant Immunol 1997;5:212-8.

4. Lin SS, Weidner BC, Byrne GW, et al. The role of antibodies in the pathogenesis of acute vascular rejection of pig-to-baboon cardiac transplants. J Clin Invest 1998;101:1745-56.

5. Auchincloss H, Sachs DH. Xenogeneic transplantation. Annu Rev Immunol 1998;16:433-70.
6. Platt JL. New directions for organ transplantation. Nature 1998;392(6679 Suppl):11-17.

7. Yeatman M, Daggett CW, Parker W, et al. Complementmediated pulmonary xenograft injury. Transplantation 1998;65:1084-93.

8. Pierson RN 3rd, Kasper-Konig W, Tew DN, et al. Hyperacute lung rejection in a pig-to-human transplant model. Transplantation 1997;63:594-603.

9. Daggett CW, Yeatman M, Lodge AJ, et al. Total respiratory support from swine lungs in primate recipients. J Thorac Cardiovasc Surg 1998;115:19-27.

10. Macchiarini P, Oriol R, Azimzadeh A, et al. Evidence of human non- $\alpha$-galactosyl antibodies involved in the hyperacute rejection of pig lungs and their removal by pig organ perfusion. $\mathrm{J}$ Thorac Cardiovasc Surg 1998;116:831-43.

11. Byrne GW, McCurry KR, Martin MJ, McClellan SM, Platt JL, Logan JS. Transgenic pigs expressing human CD59 and decayaccelerating factor produce an intrinsic barrier to complementmediated damage. Transplantation 1997;63:149-55.

12. Alvardo CG, Cotterell A, McCurry K, et al. Variation in the level of xenoantigen expression in porcine organs. Transplantation 1995;59:1589-96.

13. Falk RJ, Dalmasso AP, Kim Y, et al. Characterization of a monoclonal antibody and immunohistochemical localization in renal disease. J Clin Invest 1983;72:560-73.

14. Platt JL, LeBien TW, Michael AF. Interstitial mononuclear cell populations in renal graft rejection: identification by monoclonal antibodies in tissue sections. J Exp Meal 1982;155:17-30.

15. Platt JL, Turman MA, Noreen HJ, Fischel RJ, Bolman RM, Bach FH. An ELISA assay for xenoreactive natural antibodies. Transplantation 1990;49:1000-1.

16. Yu PB, Holzknecht ZE, Bruno D, Parker W, Platt JL. Modulation of natural IgM binding and complement activation by natural $\operatorname{IgG}$ antibodies. J Immunol 1996;157:5163-8.

17. Parker W, Bruno D, Holzknecht ZE, Platt JL. Xenoreactive natural antibodies: isolation and initial characterization. J Immunol 1994;153:3791-803.

18. Holzknecht Z, Coombes S, Blocher B, Lau C, Davis RD, Platt $\mathrm{J}$. Identification of porcine pulmonary microvascular endothelial cell antigens reactive with human serum xenoreactive natural antibodies A536 [abstract]. Transplantation 1999;67: S381.

19. Posther KE, Lau CL, Gonzalo-Gonzalez-Stawinski, et al. Xenoreactive antibody recognition of relevant antigens in swine kidney and lung (A535) [abstract]. Transplantation 1999;67:S381.

20. Ruggeri ZM. Glycoprotein Ib and von Willebrand factor in the process of thrombus formation. Annals of the New York Academy of Sciences 1994;714:200-10.

21. Pierson RN 3rd, Pino-Chavez G. Experimental lung xenografting. In: Cooper DKC, White DJG, Platt JLP, editors. Xenotranplantation: the transplantation of organs and tissues between species. Berlin, New York: Springer; 1997. p. 463-77.

22. Pierson RN 3rd, Parker RE. Thromboxane mediates pulmonary vasoconstriction and contributes to cytotoxicity in pig lungs perfused with fresh human blood. Transplant Proc 1996;28:625.

23. Holzknecht ZE, Platt JL. Identification of porcine endothelial cell membrane antigens recognized by human xenoreactive natural antibodies. J Immunol 1995;154:4565-75. 